\title{
Computational Ferrying: Efficient Scheduling of Computation on a Mobile High Performance Computer ${ }^{\underline{\pi}}$
}

\author{
Alireza K. Monfared ${ }^{\mathrm{a}, *}$, Ellen W. Zegura ${ }^{\mathrm{a}}$, Mostafa Ammar ${ }^{\mathrm{a}}$, David Doria ${ }^{1}$, David Bruno ${ }^{1}$ \\ ${ }^{a}$ School of Computer Science, Georgia Institute of Technology, Atlanta, GA \\ ${ }^{b}$ United States Army Research Laboratory, Aberdeen, MD
}

\begin{abstract}
Mobile devices are often expected to perform computational tasks that may be beyond their processing or battery capability. Cloud computing techniques have been proposed as a means to offload a mobile device's computation to more powerful resources. In this paper, we consider the case where powerful computing resources are employed on a vehicle. This vehicle can be re-positioned in real time to receive computational tasks from user-carried devices. This scenario finds application in challenged environments and may be used in a military or disaster relief setting. It is further enabled by increasing feasibility of constructing a Mobile High Performance Computer (MHPC) using rugged computer hardware with form factors that can be deployed in vehicles. By analogy to prior work on message ferries and data mules, one can refer to the use of MHPCs as computational ferrying. After illustrating and motivating the computational ferrying concept, we turn our attention into the challenges facing such a deployment. These include the well-known challenges of operating an opportunistic and intermittently connected network using message ferries - such as devising an efficient mobility plan for MHPCs and developing techniques for proximity awareness. In this paper, first we propose an architecture for the system components to be deployed on the mobile users and the MHPCs. We then focus on defining and solving the MHPC movement scheduling problem with sufficient generality to describe a number of plausible deployment scenarios. We conduct a number of experiments that provide an understanding of how the performance of the system using MHPCs is affected by various parameters.
\end{abstract}

Keywords: Opportunistic and delay tolerant networks, Mobile computing systems

\section{Introduction}

There is an ever-present cycle of software exceeding current hardware capabilities and driving future development. This trend is especially acute for mobile wireless devices, which are increasingly

This work extends on our previous submission in $9^{\text {th }}$ IEEE WoWMoM workshop on Autonomic and Opportunistic Computing (Monfared et al., 2015).

*Principal corresponding author

Email addresses: alireza@gatech.edu (Alireza K. Monfared), ewz@cc.gatech.edu (Ellen W. Zegura), ammar@cc.gatech.edu (Mostafa Ammar), david.l.doria.civ@mail.mil (David Doria), david.bruno@us.army.mil (David Bruno)

Preprint submitted to Computer Communications expected to perform demanding computational tasks. Additionally, mobile devices have capability limits, battery lifetime limits, and compute power limits that restrict their ability to perform intensive computational tasks. This gap between expectations and mobile device capability is likely to continue.

In this paper we consider the case where powerful computing resources are deployed on a vehicle. These vehicles can be repositioned in real-time to be in contact with mobile devices that require remote compute resources and where no Internet connectivity is available. This scenario finds application in challenged environments and may be

September 1, 2016 
used in military or disaster relief environments. It is further enabled by the increasing feasibility of constructing a mobile high performance computer (MHPC) using rugged computing hardware with form factors that can be deployed in vehicles. While expectations in challenged environments are often tempered, low-latency, computationally intensive applications can be important. Figure 1 shows an example of tactical HPCs used in combat vehicles which is of interest to military vendors.

There are many challenges facing the deployment of a system using MHPCs. These include the well-known challenges of operating an opportunistic and intermittently connected network using message ferries, such as devising an efficient mobility plan for MHPCs and developing techniques for proximity awareness. In addition, such a system will need to include computation offloading decision making mechanisms to be deployed by mobile users, techniques for scheduling computation on MHPCs, and a strategy for handling possible mobility of the users.

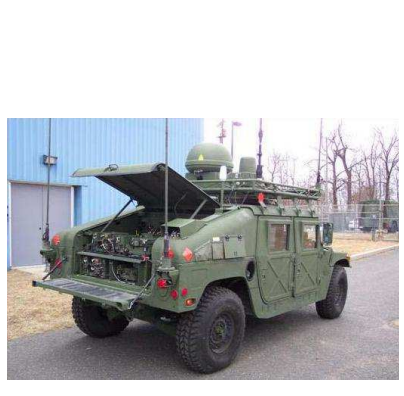

(a) HPC mounted on a(b) A sample HPC military vehicle.

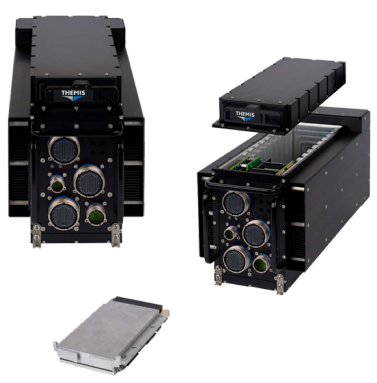
module, TSY-300X $3 \mathrm{U}$ VPX by Themis Computers Forbes (2015).

Figure 1: MHPCs are ultra-rugged, battle-proven, highperformance tactical computing solutions for combat vehicles.

In this paper, we review the related work in Section 2 , then we describe our vision of components and architecture of a system deploying MHPCs in Section 3. We then focus on the question of how to schedule the movement of the computational ferries and how to schedule task execution on their processors. To that end, we describe a model of the system in Section 4. This is followed by a formal description of the computational ferrying problem and a discussion of algorithms for deciding on the computational ferry schedule in Section 5. We then report on the results from various experiments to understand how the system performance is affected by various parameters and effects in Section 6. Finally, Section 7 concludes the work and suggests directions for future work.

\section{Related Work}

As computing devices become more powerful, expectations for performance and richness of applications continue to increase. Cloud computing techniques propose a means to offload the most intensive computation tasks from a mobile device to more powerful computing resources reached over the Internet or another network (e.g., Chun et al. (2011); Cuervo et al. (2010); Rahimi et al. (2014)). In the standard offloading scenario, the mobile device enjoys stable connectivity to the cloud, offloads some computation, and receives results back from the cloud. Variants of this scenario include local cloudlets(Satyanarayanan et al., 2009; Chen et al., 2015) that concentrate more powerful computing close to mobile devices, such as in a coffee shop, and intermittently connected clouds (Shi et al., 2012; Zhang et al., 2014) where the mobile device is assumed to have intermittent or variable connectivity to the cloud. The prior work on stationary cloudlets (Satyanarayanan et al., 2013) and placement of High Performance Computers (HPCs) (Shires et al., 2012) assumes that the powerful computing resources are pre-positioned in stationary locations.

Our work draws on and complements work in mobile cloud computing, vehicular cloud computing, and disruption tolerant networks as well as tactical high performance computing trend of work in the military.

Mobile Cloud Computing (MCC) brings the CC paradigm to the world of non-stationary devices. MCC is formally defined as "an infrastructure where both the data storage and data processing happen outside of the mobile device. 
Many forms of data processing have been shown to be advantageous if performed remotely, including image processing, natural language processing, crowd computing, sharing GPS/Internet data, sensor data applications, multimedia search, and social networking (Fernando et al., 2013). In (Cuervo et al., 2010) Cuervo et al. propose a system to offload fine-grained pieces of code that are chosen in an online manner in order to maximize the energy savings. Another example of automation of offloading is proposed by Chun et al.(Chun et al., 2011) where applications are partitioned at runtime and offloaded.

In general, the cloud need not necessarily reside on a remote Internet server. An example of this case is in Satyanarayanan et al.(Satyanarayanan et al., 2009) that concentrate on using virtual machines to instantiate the required services on more powerful computing resources close to mobile devices called cloudlets. Note that stationary cloudlets are complementary to our proposal of deploying MHPCs. Each of these approaches has its own advantages and disadvantages depending on the environment. Indeed, stationary cloudlets can be viewed as the same continuum of the $\mathrm{MH}$ PCs, where the difference is the time scale of (re)deployment.

Another line of research related to stationary cloudlets involves a network design problem which attempts to place stationary HPCs to cover the computational needs of a group of users in an area. In (Shires et al., 2012), Shires et al. investigate strategies for placement of tactical cloudlets, which are essentially parallel high performance computers (HPCs) in rough environments, to cover the computational needs of a group of users in the area (called also HPC Seeding). Finally, Zhang et al. focus on offloading algorithms considering the local load that users have and the availability of the cloudlets in an intermittently connected environment with mobile cloudlets by leveraging Markov Decision Processes (MDPs) (Zhang et al., 2014).

Tactical Cloudlet research is of interest of United States Army Research Laboratory (Shires et al., 2012; Teller et al., 2013; Li et al., 2015; Chen et al., 2015) and Department of Defense through the Software Engineering Institute (SEI) at Carnegie Melon University (Morris, 2011; Lewis et al., 2014). This latter piece of work complements our work in the sense that it places an emphasis on developing a system that discovers the computation resources in the vicinity.

The rationale for offloading computation from mobile devices to cloud or cloudlet resources has received significant attention in the literature(Chun et al., 2011; Cuervo et al., 2010; Shi et al., 2014; Li et al., 2015). The motivation for offloading computational tasks in our system derives from this general motivation. More specifically, it is desirable for a device to offload to an MHPC for computation speedup, power savings, or to have access to data stored at the server.

Our work is also related to efforts in Vehicular Cloud Computing which aims to exploit the underutilized computational resources of vehicles on streets, roadways, etc. Another, more obvious example is utilizing the computational power of the large number of cars stuck in a traffic shown in (Olariu et al., 2012). VCC emphasizes the fact that while resources on these vehicles will be used for various services, there is no designated vehicle with the responsibility to provide services to other mobile or stationary users, thus issues of distributed cooperation and coordination are central(Gerla, 2012). While we also rely on vehicles with computing or communication capacity, our work differs because we assume an MHPC with controlled mobility that is given the task of supporting other users.

Another related line of research is the vast amount of work that proposes to utilize data MULEs (Mobile Ubiquitous LAN Extensions) (Shah et al., 2003; Jea et al., 2005). In contrast to DATA MULES, our work mostly focuses on MHPCs with controlled mobility that are mostly responsible for helping nodes with computational tasks rather than data collection. The task scheduling component is also mostly absent in the data MULE line of work, while our work relies on properly scheduling the tasks on the $\mathrm{MH}-$ PCs or on the HPC. Similarly, in a delay tolerant network, Message Ferries (MF) are a set of special mobile nodes that provide communication 
services for other (possibly stationary) nodes in the network (Zhao et al., 2004). Similar to our MHPCs, which by analogy can be called "Computational Ferries", Message Ferries enjoy controlled mobility in order to serve their purpose. Usage of Message Ferries is justified in crisis scenarios where infrastructure does not exist and nodes can easily go out of range of one another, in area sensing and surveillance applications where sensors are sparsely deployed, or in scenarios where economic or privacy considerations make them a more suitable alternative (Zhao et al., 2004).

\section{System Architecture}

A system deploying MHPCs shares many of the same challenges faced by opportunistic communication systems. These challenges include concerns for accommodating various user node mobility patterns as well as using mechanisms for neighbor discovery that allow for efficient use of communication opportunities. In addition, an MHPC system, similarly to systems using message ferries (Bin Tariq et al., 2006; Zhao et al., 2004), requires careful scheduling of ferry mobility as well as efficient coordination among the ferries when more than one is used (Zhao et al., 2005). A computational ferrying system, however, possesses significant additional features that warrant special attention. These stem primarily from the fact that the MHPC is not simply delivering data but also performing computation. While data transmission is required to and from the MHPC in order to perform the computation offloading, there is an additional requirement to deliver the result when computation is completed. Furthermore, an MHPC is performing computation while moving. These features require new techniques for scheduling of movement and computation on an MHPC. We first develop an architecture for an MHPC system in this section. We focus on the MHPC scheduling problem in the remainder of the paper.

Figure 2 provides a high-level overview of the main components of the MHPC system. It consists of three components: a number of User Nodes that generate computational tasks and seek service from MHPCs; a set of MHPCs which are vehicles with a high performance computer onboard that are responsible for providing the actual service; and an MHPC Controller that manages the requests received, and dictates to $\mathrm{MH}-$ PCs their future plan for picking up, processing and delivering tasks.

Radio Environment. The three components can exchange high-level task metadata ${ }^{1}$ using a long-range, low-bandwidth radio such as those suggested in Zhao et al. (2004); Banerjee et al. (2010). A commercially available example of such radios is Xtend radio developed by Digi Inc (Inc., 2015) with ranges up to 40 miles $(65 \mathrm{~km})$. Widecoverage, low-bandwidth infrastructure using unlicensed bands may also be available and has been deployed (SIGFOX, 2015) as an alternative. This radio is assumed to be only useful for control plane operations and not capable of exchanging tasks/results. Actual tasks and results are exchanged via a short-range, high-bandwidth channel with ranges under $100 \mathrm{~m}$.

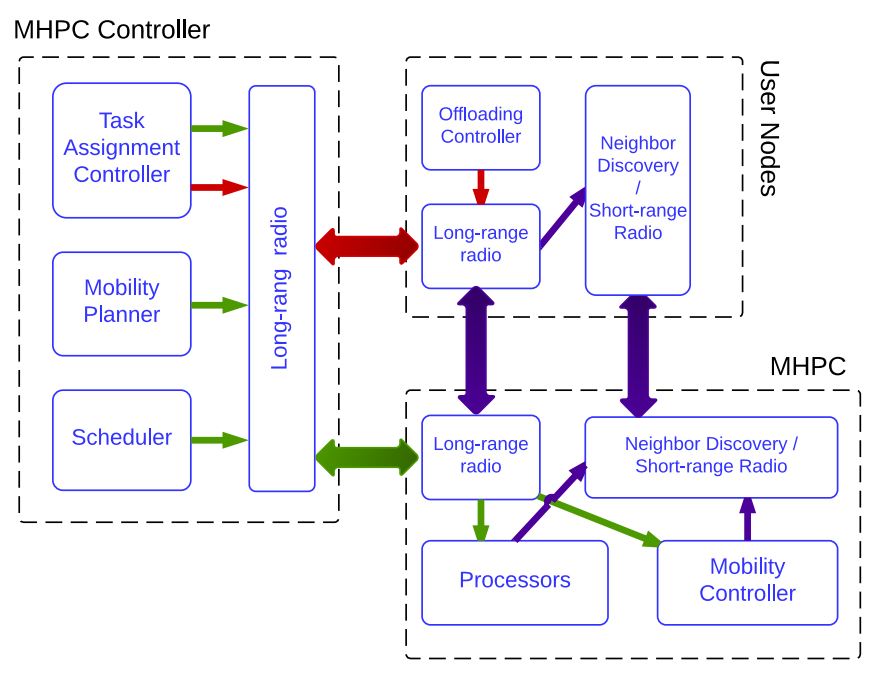

Figure 2: MHPC system architecture.

User Nodes. User Nodes are the owners of the tasks. Once a task is generated at a User Node, its Offloading Controller module decides whether the request must be sent with the task metadata to the MHPC Controller or the tasks must be

\footnotetext{
${ }^{1}$ Components of task metadata are described in detail in Section 4.
} 
processed locally. Various reasons ranging from privacy to time trade-offs may contribute to a decision to process the task locally, the details of which are outside the scope of this work. The interested reader can refer to Shi et al. (2012) for examples of work focusing on offloading decisions. Once the User Node sends out the task metadata, if it does not receive a rejection via the Longrange Radio from the controller, it will continue communicating with the assigned MHPC if necessary until the MHPC is within its range so that the task/result can be exchanged via the Shortrange Radio module.

MHPC. The MHPCs are the providers of computational service in the system. Multiple MHPCs can operate in the same area. In addition, each MHPC can have multiple processors and is able to execute several computational tasks simultaneously. The MHPCs receive their assigned tasks, mobility plan and task execution schedule from the controller via the long-range radio. The Processors of the MHPC work on the picked up tasks according to the specified execution schedule. They also report on the state of their allocated tasks to the Controller over the same radio. This state indicates how much processing of this task has been completed and what is the expected delivery time of the task. We describe in detail, in Section 5, how this information is used to dictate MHPCs their movement and the processors their execution. The Mobility Controller follows the MHPC tour indicated by the controller to visit the User Nodes for pick up and delivery. If the User Nodes are mobile, this module needs to use the long-range radio to repeatedly query the User Nodes for their current location and plan accordingly to meet them. Once the MHPC is in range with a User Node as notified by its Neighbor Discovery module, it can exchange the corresponding tasks/results to the User Nodes. Note that the MHPCs need to be equipped with Global Positioning System (GPS) to be able to go to the pick up and delivery locations.

MHPC Controller. This component handles the task offloading requests received from User Nodes. These requests are in the form of task metadata using a long-range and low-bandwidth radio. The MHPC controller uses knowledge of MHPC and User Node locations as well as their states to compute a computation and mobility schedule for the MHPCs, the components of which are detailed in Section 5.2. The computation and mobility schedule is communicated to the $\mathrm{MH}$ PCs and updated as needed via the long-range radio. Note that the MHPC Controller is a centralized unit in this architecture. To make the system more reliable, it is possible to add redundancy beyond the controller, e.g. use back up computation resources, allow multiple long range communication interfaces for the controller, etc.

Message Exchanges. In this paragraph, we review the message exchanges depicted in the arrows of Figure 2. At first the User Node sends a notification of the task metadata to the MHPC Controller using the long-range radio. Then the MHPC Controller sends a message to a chosen MHPC informing it of the task and instructing it how to pickup/process, and deliver using the longrange radio. After this, the MHPC approaches the User Node and using its short-range radio receives the task. At some later point in the time, the MHPC approaches the User Node ( or delivery Node if different) and uses the short-range radio to transfer back the processed results.

\section{System under Consideration}

We consider a system with mobile user nodes traveling in a bounded geographic area. Also present in the area are $V$ MHPCs, initially located at known locations. Each MHPC is assumed to have $m$ processors, i.e. it is capable of executing $m$ computational tasks simultaneously, one per processor. The vehicles on which MHPCs are mounted are assumed to be identical and to take a known constant time to travel between a pair of nodes as a function of locations of those two nodes $^{2}$. Many of these assumptions, and those

\footnotetext{
${ }^{2}$ Note that the constant time to travel among a pair of nodes does not necessarily imply constant speed, but in general we expect the farther the pair of nodes, the longer it takes to travel among them.
} 
that follow, can be relaxed; we keep the simpler form to make the exposition easier to follow while allowing the key insights to be highlighted.

We assume that user nodes notify the MHPC Controller of the existence of computational tasks for offloading, using a long-range, low-bandwidth radio as described in Section 3. This radio is assumed to be only useful for control plane operations and not capable of exchanging tasks/results. The user nodes are mobile, thus to facilitate task and result exchanges in the future, it is necessary to provide for meetup opportunities. In our formulation, the user node specifies a location where the MHPC can pick up the task and a location (possibly different) where the MHPC can deliver the result. Without loss of generality, we demonstrate two examples of specifications of such locations: (i) In a military setting, these locations can be considered as stationary Forward Operating Bases (FOBs) used as readily available resources for tactical missions without the need of reacquiring or moving resources. These FOBs may have inexpensive storage capabilities similar to throwboxes in Delay Tolerant Networks (Zhao et al., 2006), and while soldiers can move, they can place their tasks in their corresponding FOB after notifying the MHPC Controller and receive the results from the same or a different FOB. An example of this setting is shown in Figure 3.(ii) In a more general setting without FOBs as meeting locations, we assume there are a finite number of locations designated exclusively to facilitate task pickup and delivery. These could be chosen, for example, based on prior semantic information about the geographic field, such as safe spaces, common areas for mobile node congregation, etc.

As far as the MHPCs are concerned, tasks arrive in groups of one or more generated at the same location, the latter might happen if there is some batching before the MHPCs are notified. Each task, $i$, in a group is represented by the metadata vector $\left(R_{i}, D_{i}, T_{i}, L P, L D_{i}\right)^{3}$, where $R_{i}$ is the time that task $i$ is available for pick up at the location, $L P$, same for all tasks in the group. This

\footnotetext{
${ }^{3}$ This vector has been referred as "task metadata" in Section 3 .
}

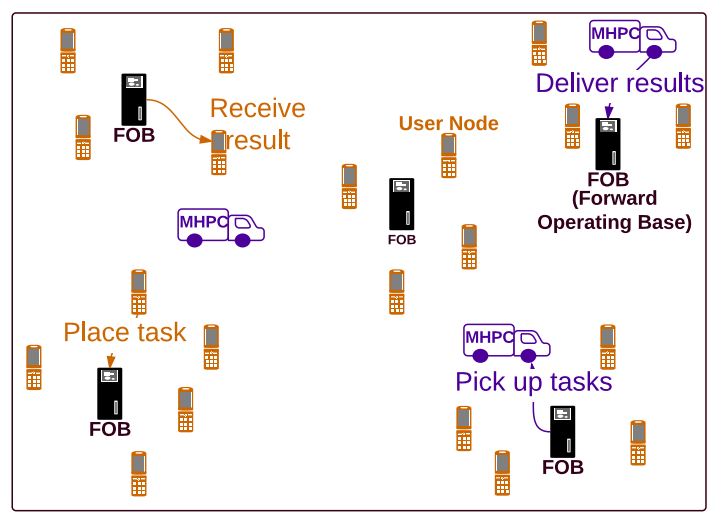

Figure 3: A framework for the MHPC problem with user nodes exchanging tasks with their FOBs, with which the MHPCs communicate for task pickup/delivery.

denotes the time taken for the user node to get to the designated location. $D_{i}$ is the deadline for the task, after which the user node is no longer interested in the result of the processing, $L D_{i}$ is the designated delivery location for the results of the processed task. In the case that $L P=L D_{i} \forall i$, we call them task location; otherwise we distinguish between a pick up location and delivery location based on designation. Finally, $T_{i}$ is the duration of time that it takes to process the task on one of the processors of an MHPC. This information is also summarized in Table 1.

\begin{tabular}{|l|l|}
\hline Parameter & \\
\hline \hline$R_{i}$ & Availability of task $i$ \\
\hline$D_{i}$ & Deadline for task $i$ \\
\hline$T_{i}$ & Time duration for task $i$ \\
\hline$L P_{i}$ & Pickup location for task $i$ \\
\hline$L D_{i}$ & Delivery location for task $i$ \\
\hline
\end{tabular}

Table 1: Components of task metadata.

After being notified of the task, the MHPC can commit to the task, in which case it will schedule the pick up, computation, and delivery of results to complete before the deadline, or it can reject the task. One interpretation of the deadlines is an estimate of the local running time of the task. In this case, the rejection of the task by MHPC means that the user node will obtain the result faster if it processes locally. Further work on offloading decisions can be found in Chun 
et al. (2011). Another interpretation is deadlines as an estimate of the remaining power on the user node's handheld device. In this case, the late delivery of the result will not be useful, since the device's battery will be depleted before the result is obtained.

Note that there is significant flexibility in our task arrival and availability formulation. For example, we can model the scenario where all tasks are immediately ready by setting all availabilities of tasks to the corresponding times when they are generated, or we can relax the deadlines by setting them to a sufficiently large value.

An MHPC can pick up a task if it visits the pick up location of the task at or after the task availability time. The MHPC travels in the region, picking up available tasks, scheduling and completing their execution on its processors, and delivering the results. As it will be described in Section 5.2, the arrival of groups of tasks affects the MHPCs' schedule, for pickups, deliveries, and processing, but does not affect its previous commitments.

Pick up and delivery of the tasks are done using the short-range, high-bandwidth radio. We assume that the transmission range of this radio is negligible compared to the dimensions of the field where MHPCs travel ${ }^{4}$.

We assume that any time required for task pick up and result delivery is negligible compared to travel and execution time. This will be true in our system because the area of a deployment is relatively large (at least few kilometers).

To illustrate the problem setting through an example, consider the simple system shown in Figure 4. An MHPC travels in the region at an average speed of $10 \mathrm{~m} / \mathrm{s}$. User Node 1 is located 5 mins drive from the current location of the MHPC, while User Node 2 is located 2 mins drive away. The user nodes are 4 mins drive apart from each other. In this simple example, the user nodes are stationary for the time period under consideration. Each user node generates a task

\footnotetext{
${ }^{4}$ If that is not the case, then a stationary HPC can be placed in a proper location where it can communicate with all user nodes
}

at time 0 . Node 1 has a task that will take 10 minutes on the MHPC, while Node 2 has a task that will take 3 minutes. Assume the processor on the MHPC allows preemption, thus a task that is started on the processor can be interrupted to allow work on another task, with resumption later from the point of interruption. The MHPC can compute and travel simultaneously. Note that the solution to such system, generated by the MHPC Controller, can be provided in run-time in general, i.e. that the controller can change decisions based on task arrivals but it cannot change any service currently given to a task neither it can change the pickup and delivery of a task once it is on an MHPC. We wil elaborate on this point further in Section 5.

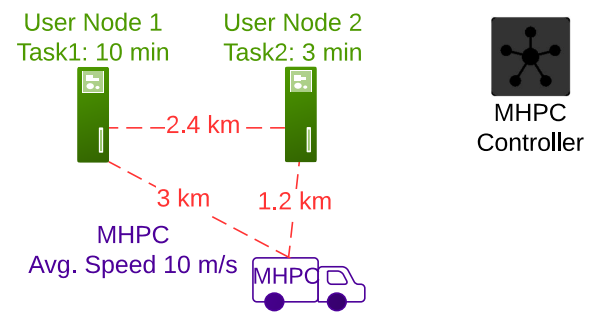

Figure 4: A simple example of MHPC scheduling problem with two nodes and a preemptive single processor MHPC.

We are interested in producing a schedule for our MHPC system that has three components:

- MHPC Assignment: This specifies the subset of tasks that are assigned to each MHPC. Each MHPC will be responsible for pick up, processing and delivery of its assigned tasks. No inter-MHPC communication is used during job processing. The tasks that are not assigned to any MHPC will be rejected since they cannot be delivered within the desired deadline.

- MHPC Tour: This is a pick up / delivery location visit schedule for each MHPC and comprises a vector of tuples in the form (location id, location visit time, location visit duration). The location visit time specifies the time that the MHPC arrives for pick up / delivery. For example, an entry $\left( \pm i, r_{ \pm i}, w_{i}\right)$ specifies that the MHPC arrives at location 
$i$ for pick up $(+)$ or delivery $(-)$ at time $r_{ \pm i}$ and waits for an amount of time $w_{i}$ to be able to perform the pickup/delivery. The location visit duration specifies how long the MHPC pauses at a location during a visit. Unlike standard traveling salesman, where this time is always 0, the MHPC may find it advantageous to arrive early and wait either for task completion or for a task to become available for pick up.

- Task Execution Schedule: This is the schedule of task execution on each processor for each MHPC. This component comprises $m$ vectors, one for each of the $m$ available processors. Each vector consists of tuples in the form (interval, scheduled task), where interval denotes the time period when the MHPC is working on the specified scheduled task. For example, an entry $\left(\left(t_{1}, t_{2}\right), i\right)$ for processor $j$ specifies that processor $j$ is working on task $i$ from time $t_{1}$ to $t_{2}$, inclusive.

We are interested in a complete schedule, defined as one in which all the tasks have been fully executed and their results delivered. There are a number of possible optimization metrics one might consider for this problem. For this paper, we focus on "normalized average flow(NAF)" as defined below for each MHPC:

$$
N A F=\max _{k \in \text { Vehicles }}\left(\underset{i \in \text { Tasks }}{\text { Average }} \frac{r_{-i}-R_{i}}{T_{i}}\right)
$$

where, $r_{-i}$ indicates the moment when the results of the task are delivered. This metric will be equal to 1 if there is a dedicated MHPC that is present at the location of each task before it is revealed. This gives us a lower bound to evaluate the algorithms of Section 5.2. We have chosen this objective value because it shows the interest of user nodes in its numerator by trying to deliver the tasks as soon as possible while being fair due to the normalization in the denominator by not prioritizing a long task over many short tasks ${ }^{5}$.

\footnotetext{
${ }^{5}$ The algorithms described in Section 5.2 are capa-
}

\section{Work Conservation and Task Preemption}

Our model for task processing on the MHPC's $m$ processors assumes no processor sharing. That is each processor is allocated at most one task at a time. We assume that all processors in the MHPC are of the same compute power. We assume that the processors are preemptive with resume, i.e., the schedule allows preemption and a task already in process may be paused in favor of another task. The preempted task is resumed at a later time.

The following lemmas capture results about preemption and work conservation:

Lemma 1: If task preemption is allowed in the MHPC scheduling then an optimal schedule is always work conserving. In a work conserving schedule the MHPC never queues a task if there are available processors.

Proof: To see this, if we discretize the time with the pick up and delivery moments, in any non-work-conserving solution, there exists at least one node $i$ for which its task is picked up at time $r_{i}$ but the CPU has been kept idle till the next pick up at some $r_{j}>r_{i}$. Since the preemption is permitted, the non-usage of the interval $\left[r, r_{j}\right]$ can account to a higher waiting time at the delivery of $i$ or a node picked up before $i$ and delivered after it, which could have been avoided (alleviated) if $i$ or that other node would have been processed in (a part of) this interval.

Lemma 2: An optimal schedule for the preemptive MHPC with resume given a specific visit order, is one that visits the accepted tasks of the MHPC in the order of delivery. Preemption might happen when a newly picked up task is scheduled to be delivered earlier.

Proof: It suffices to propose an example where a non-work-conserving schedule is optimal. Such setting is depicted in Figure 5 for a single processor MHPC . Assume that the job at user node 1 is much longer than the other two, i.e., $T_{1} \gg T$,

ble of replacing any objective value fitting other scenarios without affecting the fundamentals of the algorithm. Some other possible objective values of interest include makespan (the time that the latest MHPC finishes the processing of its last task), average completion (the average time between pick up and delivery of all tasks), and average waiting time for all tasks. 
also assume the processing time of this job is at least twice its travel time from user node 2, i.e., $T_{1}>2 t$. Finally assume that deopt node , 0 , is much closer to user node 1 than 3 , i.e., $t_{0}^{\prime} \gg t_{0}$. It can be shown that an optimal visit order is as follows:

$[+0 \rightarrow+1 \rightarrow+2 \rightarrow-2 \rightarrow+3 \rightarrow-3 \rightarrow-1 \rightarrow$ $-0]$, working out the math, the optimal schedule holds processing task at user node 1 once picked up and only starts this task, after the task at user node 3 is picked up and finished processing. Such solution is non-work-conserving and optimal with a total time of $t_{0}+t+T+t+T+T_{1}+t_{0}$.

Any work-conserving solution that processes task at user node 1 immediately after pick up can be shown to have a total time of $t_{0}+T_{1}+$ $T+t+T+2 t+t_{0}$ which is $t$ time units longer. This means that the work-conserving is not always optimal for the MHPC Scheduling Problem with non-preemptive scheduler.

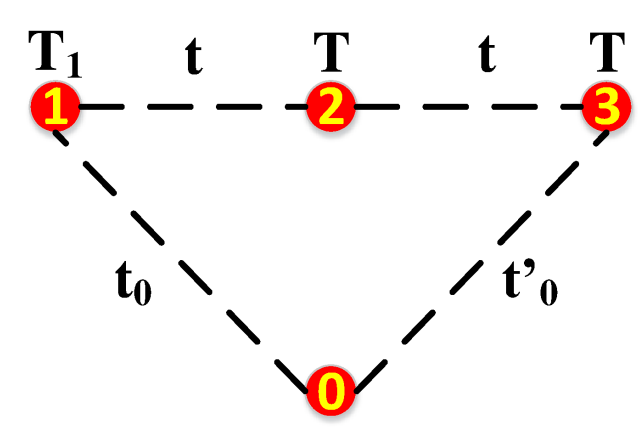

Figure 5: Comparison between work-conserving and nonwork-conserving solutions.

In the algorithms presented in Section 5.2, we use these lemmas to determine the order of processing the tasks on each MHPC.

\section{Scheduling Algorithms}

In this section, we first show how to formulate a simplified version of the MHPC scheduling problem as a Mixed Integer-Linear Program(MILP). While it is possible to solve this formulation on small instances of the problem, the MILP is not easy, or scalable, to solve larger instances of the problem. Thus, we utilize insights from the
MILP to propose scalable heuristic scheduling algorithms.

\subsection{MILP Formulation}

Before doing so, we formulate the problem as a Mixed Integer-Linear Program (MILP) that we solve on small instances to get insight into the characteristics of optimal solutions. The MILP formulation can be made very general; for exposition we present the simplified case of the problem where all of the tasks are arriving together at $t=0$ and there is a single vehicle with a nonpreemptive processor serving them.

$$
\begin{array}{cl}
\underset{x, r, w, s, y}{\operatorname{minimize}} & (\text { Objective Value }) \\
\text { subject to } & \sum_{j \in \pm \mathcal{N}} x_{j i}=1 \quad ; \forall i \in \pm \mathcal{N} \\
& \sum_{j \in \pm \mathcal{N}} x_{i j}=1 \quad ; \forall i \in \pm \mathcal{N} \\
& s_{i} \geq r_{+i} \quad ; \forall i \in \mathcal{N} \\
& s_{i}+T_{i} \leq r_{-i}+w_{i} \quad ; \forall i \in \mathcal{N} \\
& r_{-i} \geq r_{+i} \quad ; \forall i \in \mathcal{N} \\
& r_{+i}+t_{i j}-\left(1-x_{i j}\right) M \leq r_{j} \\
& \forall i \in \mathcal{N}, \forall j \in \pm \mathcal{N}, j \neq i, 0 \\
& r_{-i}+w_{i}+t_{i j}-\left(1-x_{i j}\right) M \leq r_{j} \\
& \forall i \in \mathcal{N}, \forall j \in \pm \mathcal{N}, j \neq i, 0 \\
& s_{i}+T_{i}-\left(1-y_{i j}\right) M \leq s_{j} \\
& \forall i, j \in \mathcal{N}, j \neq i \\
& y_{i j}+y_{j i}=1 \quad ; \forall i, j \in \mathcal{N}, j \neq i
\end{array}
$$

The meaning of the parameters used in the formulation are described in Table 2 and 3. Briefly, a key element of the formulation is to identify distinctly the pick up locations and delivery locations of each task $i$, denoted $+i$ and $-i$ respectively. The decision variables express the route plan via the variables $x_{i j}$ which is 1 if location $j$ is visited immediately after location $i$ and 0 otherwise. The task pickup and delivery times are expressed in the decision variables $r_{ \pm i}$. The task schedule order is captured in $y_{i j}$ and the processing start time for each task in $s_{i}$. In some scenar- 
ios the MHPC will reach the destination before the task has completed processing. In this case the waiting time at the destination before delivery is reflected in $w_{i}$.

The constraints in the formulation are generally straightforward. Tasks cannot start processing until after they have been picked up and cannot be delivered until after they have been fully processed.

\begin{tabular}{|l|l|}
\hline Parameter & \\
\hline \hline$+i$ & $\begin{array}{l}\text { Id for pick up location of } \\
\text { task } i\end{array}$ \\
\hline$-i$ & $\begin{array}{l}\text { Id for delivery location of } \\
\text { task } i\end{array}$ \\
\hline$T_{i}$ & Time duration of task $i$ \\
\hline$t_{i j}$ & $\begin{array}{l}\text { Travel time between loca- } \\
\text { tion of tasks } i \text { and } j\end{array}$ \\
\hline$M$ & A very large number \\
\hline$\pm \mathcal{N}$ & $\begin{array}{l}\text { Set of all pick up and deliv- } \\
\text { ery locations }\end{array}$ \\
\hline
\end{tabular}

Table 2: Summary of known parameters in the problem formulation.

\begin{tabular}{|l|l|}
\hline $\begin{array}{l}\text { Decision } \\
\text { Var. }\end{array}$ & Interpretation \\
\hline \hline$x_{i j}$ & $\begin{array}{l}1, \text { if task at location } j \text { is vis- } \\
\text { ited right after } i \text { and } 0 \text { oth- } \\
\text { erwise }\end{array}$ \\
\hline$r_{ \pm i}$ & $\begin{array}{l}\text { Time that task at node } i \text { is } \\
\text { picked up/delivered }\end{array}$ \\
\hline$w_{i}$ & $\begin{array}{l}\text { Waiting time at location of } \\
\text { task } i \text { before delivery }\end{array}$ \\
\hline$s_{i}$ & $\begin{array}{l}\text { Time that task at location } i \\
\text { starts processing }\end{array}$ \\
\hline$y_{i j}$ & $\begin{array}{l}1, \text { if the task at location } j \text { is } \\
\text { scheduled right after } i \text { and } 0 \\
\text { otherwise }\end{array}$ \\
\hline
\end{tabular}

Table 3: Summary decision variables in the problem formulation.

The MHPC problem is easily seen to be NPHard. A special case of the MHPC problem occurs when there is a single vehicle with a single processor and all the tasks are known in ad- vance $(A)$. A special case of $(A)$ is TSP when the the minimum travel time among every location is larger than the maximum duration of the processing of the task. Since TSP is NP-Hard, (A) and MHPC are as well.

Since the MHPC problem is NP-Hard, we need to develop efficient algorithms to solve it. On the other hand, it is obvious that at the heart of this problem is an assignment of tasks to $\mathrm{MH}-$ PCs given their commitments (similar to a binpacking problem), a pick up / delivery order planning (similar to traveling salesman) and a task scheduling problem that is solved on the processors of each MHPC. Since greedy algorithms are widely used to solve each of these problems, we adopt a similar approach to solve the MHPC problem while also accounting for the observations expressed in the lemmas.

\subsection{Algorithms}

MHPC Daemon Algorithm: In this section, we describe the algorithms that solve the described problem. In Algorithm 1 (MHPC daemon), we describe a high-level view of the operations performed by an MHPC as it is notified of the arriving tasks. Groups of tasks that are given as input are in the form of tuples $\left(t_{i}, G_{t_{i}}\right)$ where $t_{i}$ are the moments when the MHPCs are notified of the existence of the group. Each group of tasks is in the form of $G_{t_{i}}=\left\{N=n_{t_{i}},\left\{\left(R_{1}, D_{1}, T_{1}, L P, L D_{1}\right), \ldots\right.\right.$, $\left.\left.\left(R_{N}, D_{N}, T_{N}, L P, L D_{N}\right)\right\}\right\}$. Here, $n_{t_{i}}$ is the number of tasks in this group. The task feature vectors are the same as introduced in Section 3.

The MHPC Daemon Algorithm assumes that the time taken to calculate the New Solution using Algorithm 2 is negligible, otherwise, one can add this computation time as a function of the size of the problem. Our implementation allows for batching the groups of tasks in these cases. This batching will require the MHPCs to re-calculate the solution only after either a specific amount of time has passed since the arrival of the first group of newly arriving tasks or a specific number of tasks have arrived. Batching is usually only needed to avoid frequent re-calculations of the solution. 
Input: Groups of tasks: $\left(t_{i}, G_{t_{i}}\right)$, Number of MHPCs/processors per MHPC: $(V, m)$, Service period: $t_{S}$

Output: Base Solution $=$ List of tasks assigned to each MHPC: $t a s k s_{k} \forall k \in V$, visit order for each MHPC: tour $_{k}=\left\{\ldots,\left( \pm i, r_{ \pm i}, w_{i}\right), \ldots\right\} ; \forall k \in V$, Processor schedule for each MHPC: sched $_{k}=\left\{\left(\left(t_{1}, t_{2}\right), i\right)\right\} \forall k \in V$

Initialization: Use Algorithm 2 to solve for the tasks available at time 0 ;

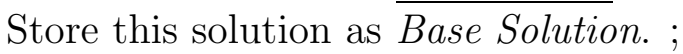

while time $<t_{s}$ do

for Each task group $\left(t_{i}, G_{t_{i}}\right)$ do

Use Cutting Algorithm to cut the solution at $t_{i}$;

Store the portion of the base solution before $t_{i}$ and store it in Base Solution. ;

Merge the portion of the solution after $t_{i}$ with the tasks in $G_{t_{i}}$;

Use Algorithm 2 to solve for the merged input;

Store this solution as New Solution;

Properly Merge New Solution into Base Solution

end

end

return Base Solution;

Algorithm 1: MHPC Daemon Algorithm.

In the next sections, we describe the underlined components of Algorithm 1.

MHPC Task Group Scheduling Algorithm: At the heart of the MHPC Daemon Algorithm lies Algorithm 2 (MHPC Task Group Scheduling Algorithm) which can solve an instance of the MHPC problem with knowledge of a group of tasks.

Note that in this specification, the objective value is an independent module. In the experiments throughout this paper, we have chosen to use the NAF as discussed in Section 3, however any other objective value that uses the information about task assignments, visit orders and the schedule can be used. For instance, if the gas consumption of the vehicles on which MHPCs are mounted is a concern, one can use a metric that gives a higher weight to the total distance traveled. Note that the outer loop for this algorithm is linear in the number V of MHPCs, but the inner loop is roughly quadratic in the number of tasks. Hence, the overall cost is $O\left(n^{2} V\right)$.

Cutting Algorithm: As described in Algorithm 1, we need to cut the solution at the moment that a new group of tasks arrive. This action involves keeping the parts of the solution on which the MHPC has already done some work and replanning for tasks that are not yet served or are partially served along with the new group of tasks. To explain the operations involved in cutting the solution at the moment $t_{i}$ when a new group of tasks arrives, assume, for the tour of $k^{\text {th }} \mathrm{MHPC}$ that $t_{k}$ is the moment that the MHPC finishes serving (delivers the result) the last task among those for which it has been planning since the last period.

- If $t_{i} \geq t_{k}$, we assume that each MHPC has stopped at the location of delivery of its last task. When planning for the group of tasks, we assume that these are the new initial locations and use Algorithm 2. All of the solution in the previous step is marked as served and Algorithm 2 solves the problem only for the newly arrived groups of tasks to deliver the New Solution.

- If $t_{i}<t_{k}$, we need to re-plan for any pick up, processing and delivery that has occurred after $t_{i}$. To do this, we need to find the location that each MHPC will be at time $t_{k}$. At this moment, the MHPC, will be either traveling between two user nodes and its location 
Input: Number of tasks: $n$, Specifications of each task $i$ : $\left(R_{i}, D_{i}, T_{i}, L P_{i}, L D_{i}\right)$, Number of MHPCs/processors per MHPC: $(V, m)$

Output: Solution $=$ List of tasks assigned to each MHPC: task $s_{k} \forall k \in V$, visit order for each MHPC: tour $_{k}=\left\{\ldots,\left( \pm i, r_{ \pm i}, w_{i}\right), \ldots\right\} ; \forall k \in V$, Processor schedule for each MHPC: sched $_{k}=\left\{\left(\left(t_{1}, t_{2}\right), i\right)\right\} \forall k \in V$

Initialization: Mark all tasks as unvisited;

Sort the tasks to examine in some order;

while there are unvisited tasks do

Take the next unvisited task, $i$;

for Each $M H P C, K$, in $V$ do

for Each placement of task i's pick up and delivery in the current visit order of the MHPC do

Schedule processing order of tasks according to an earliest delivery order first (Lemma $2)$;

Store this assignment, visit order, and schedule as Temporary Solution;

if Temporary Solution gives minimum increase in the objective value then

I Choose Temporary Solution as Solution end

end

end

end

Based on the schedule and visit order in Solution, calculate $r_{ \pm i}$ and $w_{i}$ for each pick up / delivery; return Solution;

Algorithm 2: MHPC Task Group Scheduling Algorithm.

can be found using interpolation or it will be done with serving the last task and its location will be the location of that task. To cut the solution at $t_{k}$, we shall keep any task that needs (pick-up, processing and delivery), or (partial-processing, delivery $)^{6}$ or (delivery $)^{7}$. We then add the new group of tasks to this list of non-served tasks and use Algorithm 2 to deliver the New Solution. Note that any of the tasks that remains after cutting the solution will be marked as served.

Merging Solutions: This operation merges the New Solution that results after applying Algorithm 2 with the Base Solution. This merging shall account for the offset that the moment of

\footnotetext{
${ }^{6}$ For this class of the tasks, we need to do more processing on them for whatever that has not been processed before $t_{i}$.

${ }^{7}$ This class of tasks have been processed completely, but since the original delivery plan has fallen after $t_{i}$, they will require re-planning of delivery after cutting.
}

arrival of the new tasks, $t_{i}$ imposes. This will provide a complete solution to the problem.

\section{Evaluation}

In this section, we present various sets of results to understand the proposed algorithms. We first show instances of scheduling that result from the algorithms of Section 5.2. We then show that our heuristic algorithm provides a close approximation to the performance of the schedule that results from directly solving the MILP. Next we show that a preemptive scheduler in the heart of Algorithm 2 outperforms a non-preemptive scheduler. Then in Sections 6.4 and 6.5 we run simple experiments to answer interesting questions about the algorithm. Finally, in the rest of this section, we overview the effect of various system parameters on the performance of the MHPC system. 


\subsection{Examples of the Algorithm}

Figure 6a illustrates a problem instance as well as a solution to this instance. In this scenario, there are four node locations, labeled as circles with the integers $1-4$ inside. There is one depot location, labeled as a circle with a 0 inside.

Assume that a two processor MHPC is initially parked at location 0 , and there are short and longer tasks of duration 10,20,50,100 seconds at user nodes $1,2,3$, and 4 respectively. There is a single vehicle serving these nodes moving at a constant speed of $10 \mathrm{~m} / \mathrm{s}$ reasonable for a combat vehicle. Distances are proportional to the linear path from one node to another.

Figures $6 \mathrm{a}$ and $6 \mathrm{~b}$ show the tour component of the solution to the problem described above using a non-preemptive and a preemptive scheduler respectively. The tour specifies visit times, $r_{+i}$ for pick up and $r_{-i}$ for delivery, and visit durations, in the form of waiting time before delivery, $W_{-i}$. The order in which nodes are visited is indicated by numbers on the arcs.

To complete this example, we have shown the schedule component of the solutions in Figure 6c. Since we have a single vehicle, there is no assignment component.

Focusing on the non-preemptive case, the MHPC starts by visiting node 4, picking up the task and beginning to work on it while traveling to node 3 .

When the MHPC arrives at node 3, it picks up the task but does not begin working on it even though there is a processor available. Instead, the MHPC travels to node 2, picks up its task, schedules it on the second processor, and remains at node 2 until that task completes (indicated by the self-arc at node 2). Once the task at node 2 is completed and delivered, the MHPC assigns task 3 to the second processor and moves back to node 4 . Upon arrival at node 4 , task 4 has been completed and can be delivered. The MHPC then moves back to node 3 to deliver its finished task, and then visits node 1 to pick up, process, and deliver its task. Finally, the MHPC returns to the depot.

The preemptive case begins in the same way, but unlike the non-preemptive case, it schedules task 3 on the second processor as soon as task 3 is picked up. It then travels to node 2 and picks up its task, preempts task 4 on the first processor, and waits at node 2 to complete and deliver its task. The MHPC then restarts task 4 on the first processor and returns to node 3 to deliver the task that has completed. The MHPC travels to node 1 to pick up, process and deliver its task. At the point of arrival at node 1, both processors are available; the first processor is chosen for task. The MHPC then travels back to node 4 to deliver its task and then returns to the depot. The preemptive case uses the processors more efficiently and is able to reduce the amount of time spent traveling without doing any processing. The nonpreemptive solution takes 211 seconds while the preemptive solution takes 198 seconds.

Also note that following the work-conserving and non work-conserving properties discussed in Section 4, we can see that, for example, the MHPC decides to postpone the processing of task at node 3 , even after arrival at this node, so that it can provide immediate processing to task at node 2 that is to be met later.

\subsection{Heuristic Validation}

We can compare the constructive heuristic with the exact solution from our formulation of the problem in Section 5.1 for small instances. We use the scenario shown in Figure 6a with a nonpreemptive scheduler, a single processor MHPC and all tasks available at the start.

We keep all four tasks equal in length and examine the solutions found as we change the task time duration from $1 s$ to $100 \mathrm{~s}$. Figure 7 shows the performance of the heuristic and the exact solution; the solutions have similar performance over the task time range, with a small advantage for the exact solution for tasks of duration 2040 seconds. Small tasks are generally handled by visiting a node, waiting to complete the task, and delivering the result. Middle values of task duration are most challenging since they typically require schedules that move and compute at the same time. After the task duration exceeds 53 seconds, the performance of both solutions is the same and linearly increasing with task duration. 


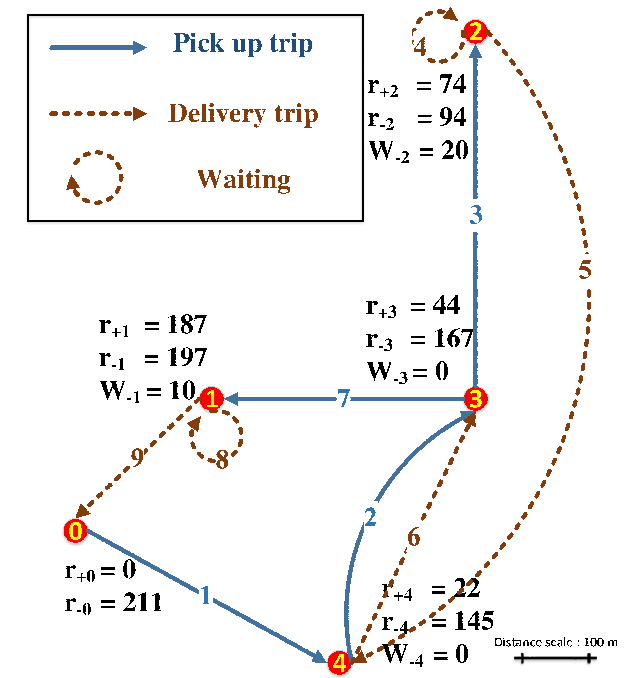

(a)

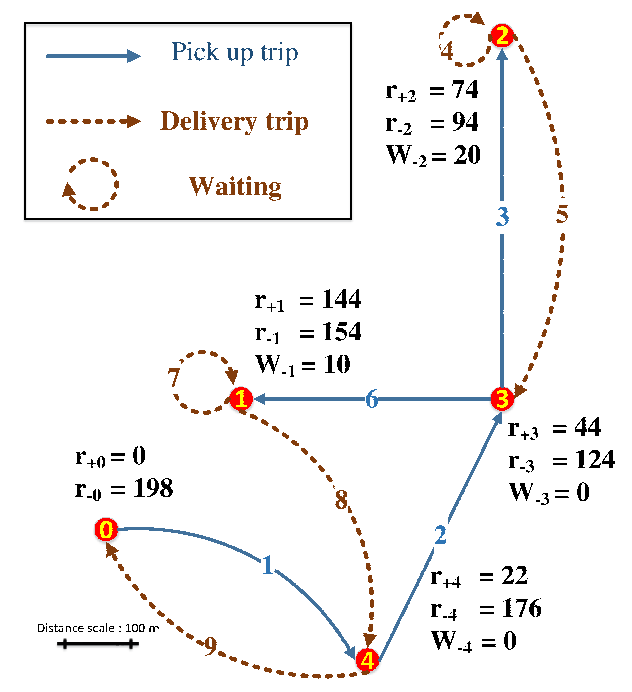

(b)

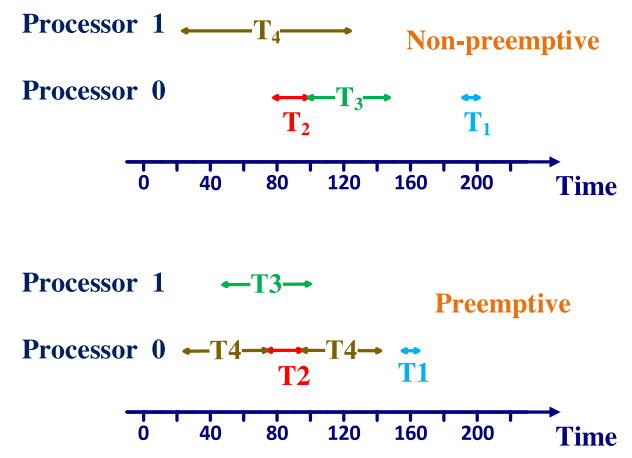

(c)

Figure 6: Tour and schedule component of a sample solution of a problem of a two processor MHPC serving 4 user nodes. Tour starts from location 0 and can be followed using the numbered arrows. $r_{+i}, r_{-i}, W_{i}$ indicate the pick up, delivery, and waiting times at user node $i$. Figure (a) describes the tour in the case of a non-preemptive and Figure (b) describes the same for a preemptive schedule. Figure (c) shows the schedule in both case; each schedule shows intervals that either of the two processors are busy processing a task. All time values are in units of seconds.

Once tasks become long, the time to process the tasks becomes the determining factor in completion time, rather than travel time.

For the single processor, single vehicle, nonpreemptive example this transition point occurs when the time to return to depot exactly equals to $\min _{i} t_{0 i}+\min _{i} t_{0 i}+\sum_{i=1}^{n-1} T_{i}$, where $\min _{1}$ and $\min _{2}$ are the first and the second minimum of the given expression.

We have repeated this experiment for various small examples and for multiple processors, and we generally observe a close agreement between the exact solution and the heuristic. We expect the gap between exact and heuristic solutions to be larger for bigger examples where the greedy algorithm can make a mistake that propagates to

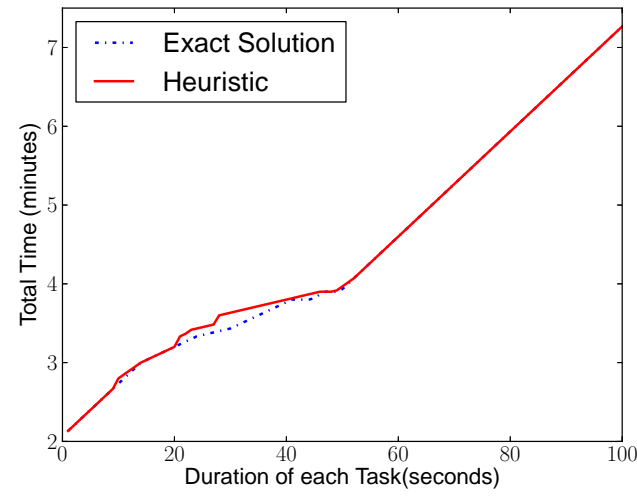

Figure 7: Comparison of the exact solution and the heuristic for a non-preemptive single processor MHPC serving 4 user nodes.

produce a solution further from exact. 


\subsection{Effect of Preemption}

We next explore the effect of allowing preemption as we increase the number of processors on the total completion time to explore if any benefits can be gained by preempting the schedule in multiple processor cases. Note that for the simulations in this section and all upcoming sections, we have used a Python-based framework that calculates the vehicle mobility and processing schedules. Interested readers can contact the authors for the source code.

In this experiment, we use 25 nodes, plus a single depot and a single MHPC, deployed in a $10 \mathrm{~km} \times 10 \mathrm{~km}$ field. We face a challenge in determining what models to use for node geographic distribution and task time distribution, since $\mathrm{MH}$ PCs are not yet in significant use. We make a choice that produces some interesting options for the heuristics.

Specifically, we use a clustered Gaussian model (CGM) for node distribution, which clusters nodes around hotspots according to a Gaussian distance distribution. We manually place four hotspots reasonably far from each other in the field. We force the model to spread nodes so that no two are within $100 \mathrm{~m}$ of each other by simply creating enough number of scenarios until one conforms with the minimum inter-node distance. Nodes that are very close, e.g., within radio range, can be treated by the heuristic as essentially the same node with multiple tasks.

We derive the duration of the tasks from a one-dimensional Clustered Gaussian model, with means around the values of 10, 25, 50, 100, 200, $600,800,1200$ and 2000 seconds. This guarantees that a variety of short and long tasks are present in the experiment.

Finally, we derive the inter-arrival times between the availability of each of the tasks from an exponential distribution with a beta parameter of 10

We vary the number of processors from 1 to 10 and observe the return time of the vehicle to the depot for the improved version of non-preemptive and preemptive heuristics. Figure 8 shows performance for both experiments averaged over 20 runs. We have measured the confidence interval for the first few experiments, but they are omitted from the figures for clarity and since they were acceptable and low. The non-preemptive version benefits most substantially from an increase in number of processors, reducing completion time to roughly half for two processors as compared to one and again to roughly one-third for three processors as compared to one.

Both non-preemptive and preemptive see a saturation in performance improvement as the number of processors reaches 7-8, a value tied to the specifics of the node geographical layouts and task times.

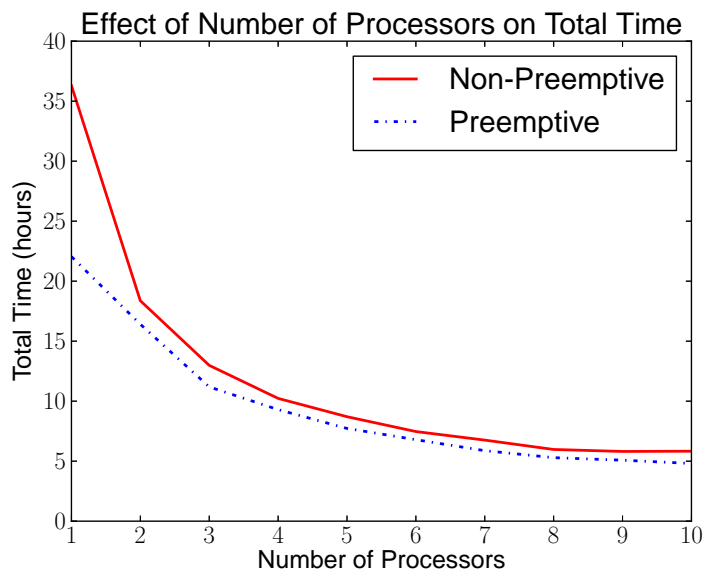

Figure 8: Effect of number of processors.

\subsection{Behavior of the Algorithm with Distance}

We start the evaluation section with some simple tests designed to understand the behavior of the algorithm. The baseline for these tests are as follows: task locations are chosen to be at the center of one of the 8 numbered zones in the $5 \mathrm{~km} \times 5 \mathrm{~km}$ field shown in Figure 9 . Pick up and delivery locations of each task are the same, aka task locations. Single processor MHPCs move with an average speed of $10 \mathrm{~m} / \mathrm{s}$. All task durations are the same and they are immediately available after the MHPC is notified and do not have a deadline. Tasks arrive in groups of 1 generated by a Poisson process. For the experiments that follow we generate 600 tasks with a 1000 second mean inter-arrival time. Note that the interarrival time between these notifications shall not exceed the system capacity, i.e. tasks shall arrive 
in a way that the MHPCs can eventually serve them; otherwise delivery time of results will keep increasing and the system cannot serve the tasks. A very rough experimental estimate for a proper arrival rate is $\frac{1}{\left(n_{i} / V\right) T_{i}+d / \nu}$ where $n_{i}$ is the average number of tasks in each group that arrives, $V$ is the number of MHPCs, $T_{i}$ is the average duration of the tasks, $d$ is the dimension of the larger side of the rectangular field, and $\nu$ is the average speed of the MHPCs. Arrival rates lower than this number will result in MHPCs serving the tasks with less delay.

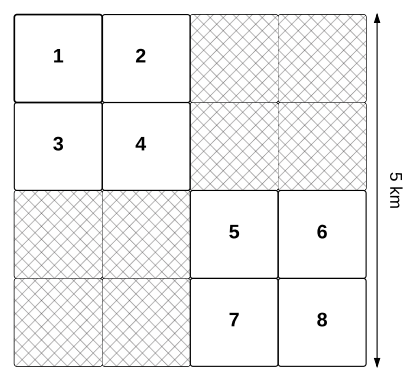

Figure 9: The baseline locations.

The first experiment investigates the effect of the distance between task locations on the performance of the algorithm. We assume a single processor MHPC $(\mathrm{m}=1$ and $\mathrm{V}=1)$ serving the baseline system, initially placed at the center of zone 1. Tasks are generated at two locations. One of the locations is always at the center of zone 1 , the other location is located at center of zones 8, 5, 4, and 1 respectively ${ }^{8}$. We repeat the tests 10 times and for three task durations: small tasks of 30 seconds, medium tasks of 300 seconds, and large tasks of 1000 seconds duration.

As Figure 10 suggests, making the locations of task generation farther apart always results in worse performance as the MHPC has to travel longer distances. This difference is more evident in the case of smaller tasks, as the MHPC will process the tasks right after pick up and going back and forth between two far locations degrades its performance. It is also notable to see that the

\footnotetext{
${ }^{8}$ In the case that both task locations are at the center of zone 1, we move each about 150 meters from the center diagonally to keep two distinct locations.
}

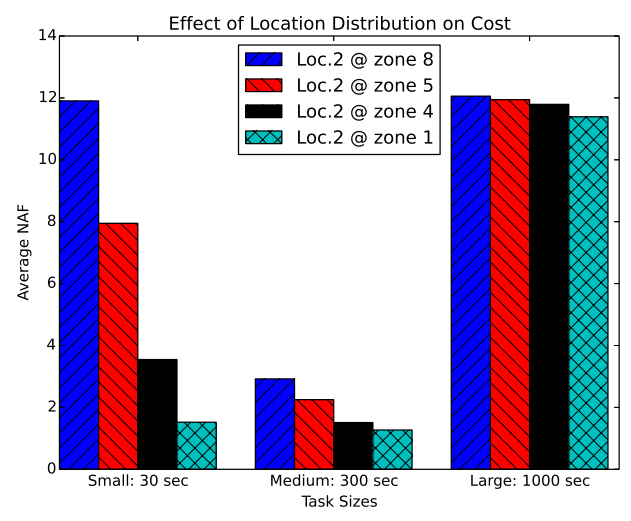

Figure 10: Effect of distance on algorithm performance.

value of NAF is smallest for medium tasks. To understand why, one shall notice that the cost for small tasks are higher, because of the normalization by the task duration which is essential for fairness when considering mixture of tasks. For larger tasks the time difference from availability to delivery is much larger compared to task durations and this in turn degrades the performance. This also shows that the MHPCs best serve medium sized tasks, with durations around half the time to travel the diameter.

\subsection{MHPC Mobility Pattern}

In this section, we perform another simple experiment to understand how the MHPCs move. To do this, consider the same setting of Section 6.4 with two locations for tasks to be generated. First location, where tasks are generated is kept to be location 1 across all experiments while the second location is changed to be placed at zones 8,5 , and 4 in three experiments. We place two single processor MHPCs, one at each location. We call the zone where the initial location of each MHPC is its "home". We then track the percentage of task pick ups done by each MHPC at its home zone. Figure 11 shows the percentage of task pick ups done by the second MHPC that are in its home zone. This behavior is symmetric for the other MHPC. It shows that for short tasks, the MHPCs always remain in the zone where they are initially placed. As the task size grows though, each MHPC may move to the other zone as well. This is, again, due to the fact that when the MH- 
PCs are busy processing a task, they will use the power of computation while moving to pick up tasks even in the other zone. While for short tasks, deliveries are mostly immediately followed by pick ups.

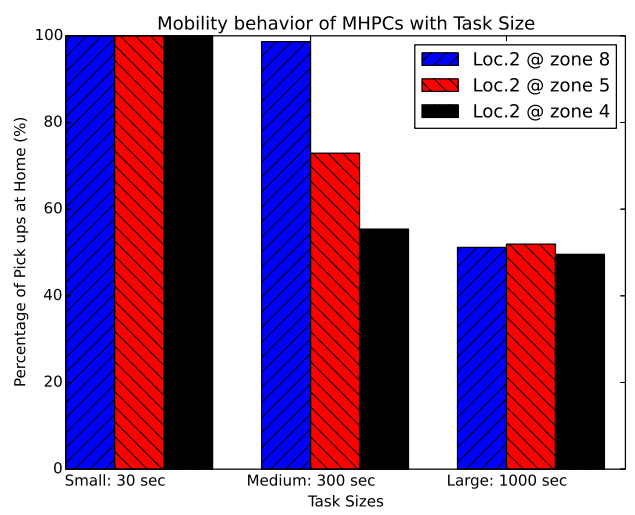

Figure 11: Mobility behavior of MHPCs with Task Size.

\subsection{Effect of Task Arrival Frequency}

In this section, we study the effect of the frequency of task arrival on the system. We turn into a more diverse model for instance generation in this case. To disperse the task locations, we divide the area into zones, as seen in Figure 9, some of these zones correspond to the areas in the field that users cannot access, e.g. unreachable mountains, areas with severe weather, contaminated areas, etc. To account for this, we divide the field into zones and with some probability (set to $80 \%$ in our experiments), we mark each zone as usable. For each usable zone, we disperse a number of locations, drawn from a uniform distribution with a mean of $n_{z} \simeq \frac{n}{\left|Z_{u}\right|}$, where $n$ is the total number of task locations and $\left|Z_{u}\right|$ is equal to the number of usable zones. In all the evaluations that will follow, we assume that there are 10 such task locations.

We run the system with 3 dual-processor $\mathrm{MH}-$ PCs serving 600 tasks arriving over time. Each task has a duration around one of the cluster centers of 30,60, 120, 240,360,600, 900, and 1200 second plus a small Gaussian error. This represents various job types that MHPCs have to deal with. Tasks are available for pick up after notification at a time exponentially distributed with a mean of 60 seconds and they do not have deadlines. We run the test for MHPCs moving with speeds of $1,2,5,10$, and $20 \mathrm{~m} / \mathrm{s}, 20$ times for each speed and report averages. We change the expected interarrival time of tasks from a minimum value respecting the capacity of the system to 3500 seconds in 500-second steps. ${ }^{9}$.

Figure 12a shows the average NAF value for various speeds. It shows that both increasing the speed and increasing the inter-arrival time of tasks decreases the cost. However, in the latter one of the MHPCs have enough time to serve all the tasks that arrive before new tasks arrive, their behavior and thus the cost remains constant. Figure $12 \mathrm{~b}$ shows the utilization of all MHPCs, defined as average time that one MHPC is using its processors. The value of the utilization is the same for all speeds, since task durations are about the same and the only difference in behavior of $\mathrm{MH}$ PCs under various speeds is that at higher speeds there is more idle time and less travel time while it is vice versa for lower speeds. The utilization however decreases as tasks arrive less frequently in all cases due to the fact that there is more idle time for the MHPCs. These figures collectively show that while having less frequent tasks allows the MHPCs to serve them better, this will result in more resources being wasted.

\subsection{Effect of Number of MHPCs and Processors}

In this section, we investigate the effect of number of the MHPCs and processors. We started the experiments in this section with settings similar to Section 6.6, changing the number of MHPCs and in another experiment the number of processors of each MHPC from 1 to 10 . We have omitted these results for the sake of space and encourage the interested reader to review them in the thesis K Monfared (2016) based on this work. They indicate that increasing the number of MHPCs or processors will decrease the cost as well as the utilization. It was noted that after a point, while there is not much decrease in the cost, utilization

\footnotetext{
${ }^{9}$ The minimum arrival rate used for the speeds are one task every $1000,500,250,150$, and 150 seconds respectively for lower to higher speeds
} 


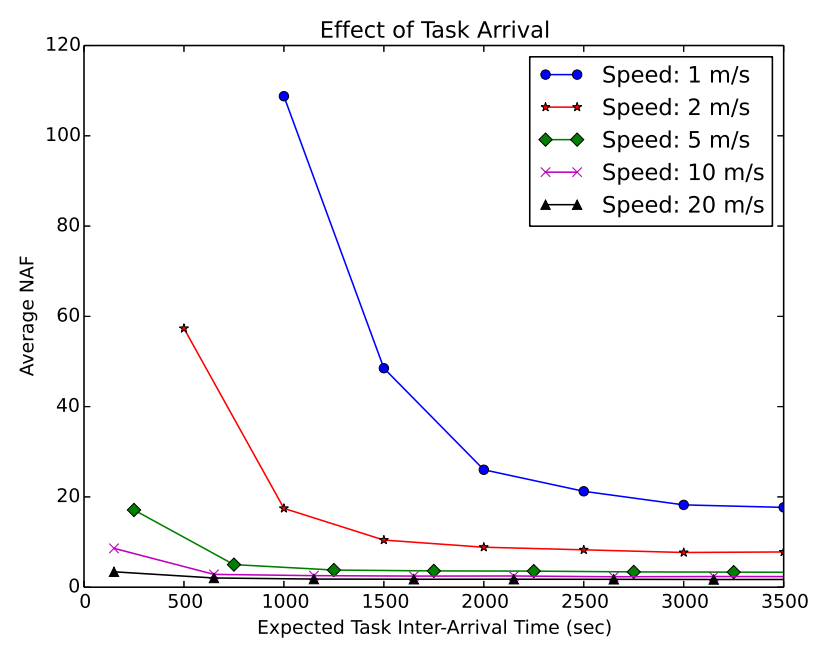

(a) Average Objective Value.

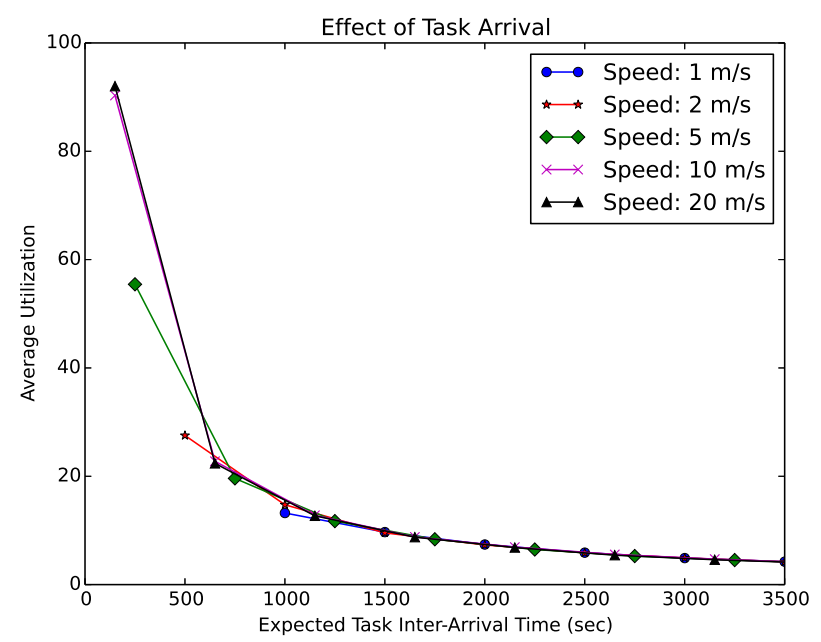

(b) Average Utilization.

Figure 12: Effect of arrival rate of tasks.

continues to decrease. This indicates that while increasing the number of MHPCs and processors are generally helpful, increase after a point will only waste resources and not provide better service.

Instead of this obvious experiment, we decided to answer a more interesting question: "how does an increase in the number of vehicles compare to an increase in the number of processors?". An answer to this question can be insightful in terms of designing the system, when one can choose between mounting a second or better HPC on the same vehicle or obtaining a new vehicle with a similar HPC.
To demonstrate this, we placed 8 task locations in the 8 shown zones of Figure 9 while the rest of the settings are similar to those of Section 6.4. For each of the task sizes, we compare three scenarios: 1 MHPC with 4 processors, 2 MHPCs with 2 processors each, a single MHPC with 4 processors. While the compute power of these three cases are equal, their mobility is different.

Figure 13 shows that going from 1 MHPC to 2 MHPCs gives considerable improvements in cost, especially when tasks are small or medium size. Dedicating 4 single processor MHPCs does not give comparable improvements compared to 2 dual processor MHPCs. The reason is that in this experiment the task locations $1,2,3,4$ can be considered one cluster and the locations 5, 6,7,8 can be considered another. Having one MHPC serving each cluster can improve the performance but two MHPCs serving the same cluster will not bring significant improvements. It is also noted that these improvements are more evident for small and medium task sizes due to the fact that with larger tasks most of the travels are done while the MHPC is processing a task and this dominates the time taken to travel for pick up and deliveries. Because of this, we suggest that designers of MHPC-based systems consider employing a strategy of initially positioning MHPCs to serve geographic clusters of mobile users.

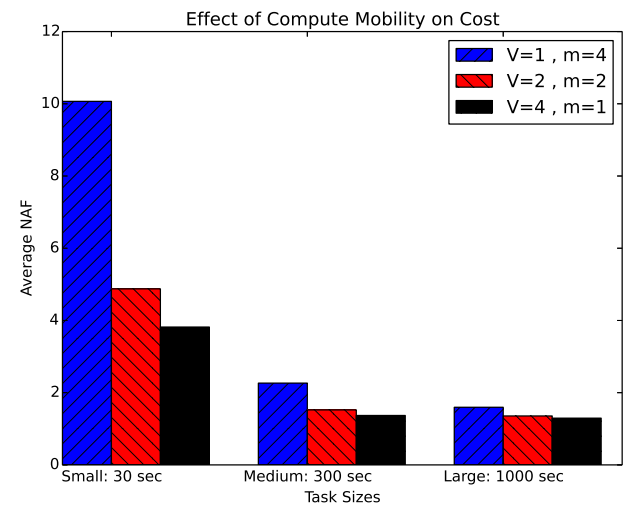

Figure 13: Effect number of MHPCs $(V)$ vs number of processors per HPC $(m)$.

\subsection{Effect of Size of Groups of Tasks}

In this section, we study the effect of the size of groups of tasks as they arrive. Such a case might 
happen if, for example, the FOBs perform some internal batching to inform the dual-processor MHPCs only after a certain amount of time or the collection of a certain number of tasks. To respect the system capacity, arrival rates are chosen to be 10000, 5000, 3000, 2000, and 1500 for speeds of $1,2,5,10$, and $20 \mathrm{~m} / \mathrm{s}$ respectively. All other settings are similar to Section 6.6. We change the size of the task groups from 1 to 9 tasks.

As Figure 14 shows, it is observed that with larger groups, there is more load on the MHPC, making it less effectively serving the tasks while utilizing more of its processing power.

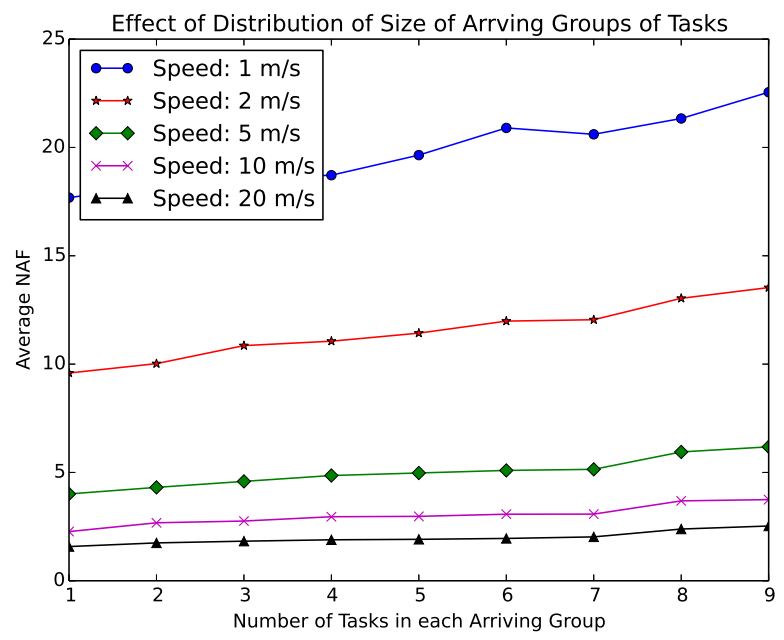

(a) Average Objective Value.

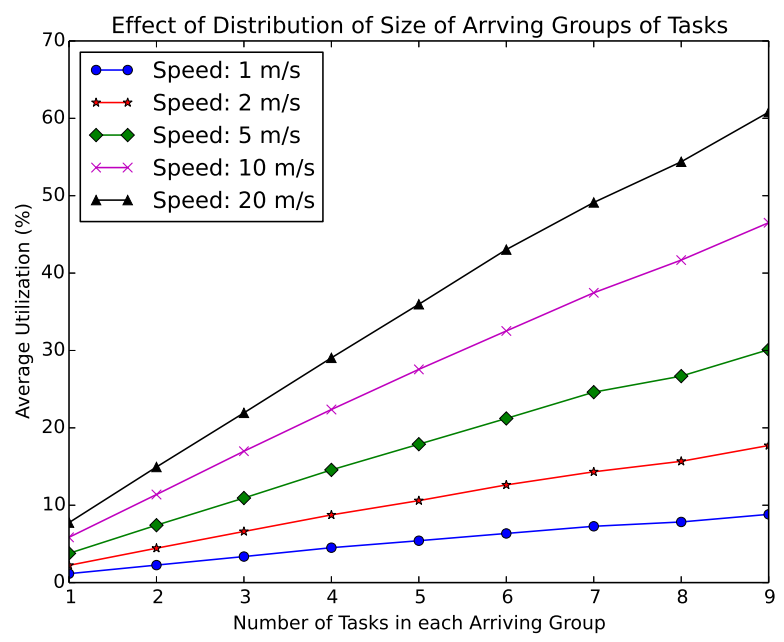

(b) Average Utilization.

Figure 14: Effect of size of the group of arriving tasks.

$\mathrm{T}$

\subsection{Effect of Deadlines}

In this section, we study the effect of deadlines. A standard test will be to utilize a scenario similar to Section 6.6 and vary "deadline margin" to see how it affects task rejection, as well as cost. To be fair among different task durations, we define deadlines for each task as "task availability + task duration + deadline margin". We change this margin in 500 increments until it is 7500 seconds. Figure 15a shows that in such a test, with higher deadline margin, there are fewer rejected tasks since the dual processor MHPCs will have more time to deliver results by the given deadline. Figure 15b shows that while the cost initially increases, when there are fewer task rejections, the cost remains steady, as the MHPC will deliver the same service and the extra deadline margin will not be helpful. Note that the reason that in the region of very small margins the cost for speeds of 1 and 2 are better than speed of 5 is due to the fact that in these small margins the high percentage of rejected tasks by the slower MHPCs results in a better service to the few remaining tasks; obviously this does not mean that the system is giving better service with respect to all tasks though. These figures show that while some deadline margin will help the MHPCs to serve the tasks instead of rejecting them, there is no necessity for very large margins.

A final experiment with the deadlines uses a simpler setting, like that of Section 6.4. Having two task locations at the center of zones 1 and 8 and all the task being of the same duration served by a single processor MHPC. As Figure 15c shows, even with a fair margin, task rejections happen more in the case of long tasks than short tasks. This is due to the fact that in general the service for larger tasks is of lower quality resulting in more deadlines being missed and more task rejections.

\section{Conclusions and Future Work}

Motivated by computational offloading requirements in mobile cloud computing, we have explored in this paper the architecture of a computational ferrying scheme where mobile high per- 
formance computers (MHPCs) provide compute resources to wireless user nodes. We studied the problem of scheduling the MHPCs' movement and processing. We started by modeling and formulating the design of such schedules as an optimization problem. We have discussed the challenges of this type of system and have developed algorithms that can be used to produce schedules for larger scale problems. We performed an extensive set of experiments to demonstrate applicability of the algorithms and show the effect of various system parameters on the performance of such a computational ferrying scheme.

This work represents a first step towards the design and evaluation of the operation of an MHPCbased mobile cloud computing system. There are a number of interesting future directions which include:

- Understanding the robustness of an MHPC system to malfunctioning of its MHPCs and how to design failure recovery mechanisms.

- Enhancements to the model to include variations such as processor-sharing scheduling on the MHPC and the possibility of picking multiple pieces of a computation from different locations or delivery to multiple user nodes.

- The consideration of communication among MHPCs either using user nodes as relays or direct exchange of information. This will no doubt draw on work on multiple message ferry scheduling (e.g., Zhao et al. (2005)).

\section{Acknowledgments}

We would like to thank the anonymous reviewers for their insightful feedback. This work was supported in part by the US National Science Foundation through grant CNS 1161879 and United States Army Research Laboratory through grant No. 36566CB.

\section{References}

Nilanjan Banerjee, Mark D. Corner, and Brian Neil Levine. Design and Field Experimentation of an Energy-Efficient Architecture for DTN Throwboxes.
IEEE/ACM Transactions on Networking, 18(2):554567, April 2010.

Muhammad Mukarram Bin Tariq, Mostafa Ammar, and Ellen Zegura. Message ferry route design for sparse ad hoc networks with mobile nodes. In Proceedings of the 7th ACM international symposium on Mobile ad hoc networking and computing, pages 37-48. ACM, 2006.

Min Chen, Yixue Hao, Yong Li, Chin-Feng Lai, and Di Wu. On the computation offloading at ad hoc cloudlet: architecture and service modes. Communications Magazine, IEEE, 53(6):18-24, 2015.

Byung-Gon Chun, Sunghwan Ihm, Petros Maniatis, Mayur Naik, and Ashwin Patti. Clonecloud: elastic execution between mobile device and cloud. In Proceedings of the sixth conference on Computer systems, pages 301-314. ACM, 2011.

Eduardo Cuervo, Aruna Balasubramanian, Dae-ki Cho, Alec Wolman, Stefan Saroiu, Ranveer Chandra, and Paramvir Bahl. MAUI: making smartphones last longer with code offload. In Proceedings of the 8th international conference on Mobile systems, applications, and services, pages 49-62. ACM, 2010.

Niroshinie Fernando, Seng W Loke, and Wenny Rahayu. Mobile cloud computing: A survey. Future Generation Computer Systems, 29(1):84-106, 2013.

David Forbes. TSY-300X 3U VPX, 8-Slot Preconfigured System, high performance computers (HPCs). Themis Computer, 2015. URL \url\{http://www . themis.com/ product/tacticalsystems/tsy300x\}.

Mario Gerla. Vehicular cloud computing. In Ad Hoc Networking Workshop (Med-Hoc-Net), 2012 The 11th Annual Mediterranean, pages 152-155. IEEE, 2012.

Digi Inc. Xtend 900mhz rf modems. XTend Radio, 2015. URL Jurl\{http://www.digi.com/products/xbeerf-solutions/modems/xtend-900mhz-rf-modems\}.

David Jea, Arun Somasundara, and Mani Srivastava. Multiple controlled mobile elements (data mules) for data collection in sensor networks. In Distributed Computing in Sensor Systems, pages 244-257. Springer, 2005.

Alireza K Monfared. Tactical HPC: Scheduling High Performance Computers in a Geographical Region. $\mathrm{PhD}$ thesis, School of Electrical and Computer Engineering, Georgia Institute of Technology, North Ave, Atlanta, GA 30332, 12016.

Grace Lewis, Sebastián Echeverría, Soumya Simanta, Ben Bradshaw, and James Root. Tactical cloudlets: Moving cloud computing to the edge. In Military Communications Conference (MILCOM), 2014 IEEE, pages 14401446. IEEE, 2014.

Bo Li, Yijian Pei, Hao Wu, and Bin Shen. Heuristics to allocate high-performance cloudlets for computation offloading in mobile ad hoc clouds. The Journal of Supercomputing, pages 1-28, 2015.

Alireza Monfared, Mostafa Ammar, Ellen Zegura, David Doria, and David Bruno. Computational ferrying: Challenges in deploying a mobile high performance com- 
puter. In World of Wireless, Mobile and Multimedia Networks (WoWMoM), 2015 IEEE 16th International Symposium on a, pages 1-6. IEEE, 2015.

Edwin Morris. A new approach for handheld devices in the military. SEIBlog.http: //blog. sei. cmu. edu/post. cfm/a-new-approachfor-handheld-devices-in-the-military, 2011.

Stephan Olariu, Tihomir Hristov, and Gongjun Yan. The next paradigm shift: from vehicular networks to vehicular clouds. Basagni, S. and Conti, M. and Giordano, S. Stojmenovic, I),(Eds), Mobile Ad hoc networking: the cutting edge directions, Wiley and Sons, New York, 2012.

M Reza Rahimi, Jian Ren, Chi Harold Liu, Athanasios V Vasilakos, and Nalini Venkatasubramanian. Mobile cloud computing: A survey, state of art and future directions. Mobile Networks and Applications, 19(2):133143, 2014.

Mahadev Satyanarayanan, Paramvir Bahl, Ramón Caceres, and Nigel Davies. The case for vm-based cloudlets in mobile computing. Pervasive Computing, IEEE, 8 (4):14-23, 2009.

Mahadev Satyanarayanan, Grace Lewis, Edwin Morris, Soumya Simanta, Jeff Boleng, and Kiryong Ha. The role of cloudlets in hostile environments. IEEE Pervasive Computing, 12(4), 2013.

Rahul C Shah, Sumit Roy, Sushant Jain, and Waylon Brunette. Data mules: Modeling and analysis of a three-tier architecture for sparse sensor networks. Ad Hoc Networks, 1(2):215-233, 2003.

Cong Shi, Vasileios Lakafosis, Mostafa H Ammar, and Ellen W Zegura. Serendipity: Enabling remote computing among intermittently connected mobile devices. In Proceedings of the thirteenth ACM international symposium on Mobile Ad Hoc Networking and Computing, pages 145-154. ACM, 2012.

Cong Shi, Karim Habak, Pranesh Pandurangan, Mostafa Ammar, Mayur Naik, and Ellen Zegura. Cosmos: computation offloading as a service for mobile devices. In Proceedings of the 15th ACM international symposium on Mobile ad hoc networking and computing, pages 287 296. ACM, 2014.

D Shires, B Henz, S Park, and J Clarke. Cloudlet seeding: Spatial deployment for high performance tactical clouds. In Parallel and Distributed Processing Techniques and Applications, 2012.

SIGFOX. White paper: M2m and iot redefined through cost effective and energy optimized connectivity. Technical report, 425, rue Jean Rostand, 31670 Labege FRANCE, 2015. URL www.sigfox.com.

Patricia Teller, Michael McGarry, Dale Shires, Song-Jun Park, Lam Naguyen, and Joseph Deroba. Enabling battlefield decision making in the tactical cloud. In Army High Performance Computing Research Center, 2013.

Yang Zhang, Dusit Niyato, Ping Wang, and Chen-Khong Tham. Dynamic offloading algorithm in intermittently connected mobile cloudlet systems. In Communications (ICC), 2014 IEEE International Conference on, pages 4190-4195. IEEE, 2014.

Wenrui Zhao, Mostafa Ammar, and Ellen Zegura. A message ferrying approach for data delivery in sparse mobile ad hoc networks. In Proceedings of the 5th ACM international symposium on Mobile ad hoc networking and computing, pages 187-198. ACM, 2004.

Wenrui Zhao, Mostafa Ammar, and Ellen Zegura. Controlling the mobility of multiple data transport ferries in a delay-tolerant network. In INFOCOM 2005. 24th Annual Joint Conference of the IEEE Computer and Communications Societies. Proceedings IEEE, volume 2, pages 1407-1418. IEEE, 2005.

Wenrui Zhao, Yang Chen, Mostafa Ammar, Mark Corner, Brian Levine, and Ellen Zegura. Capacity enhancement using throwboxes in dtns. In Mobile Adhoc and Sensor Systems (MASS), 2006 IEEE International Conference on, pages 31-40. IEEE, 2006. 


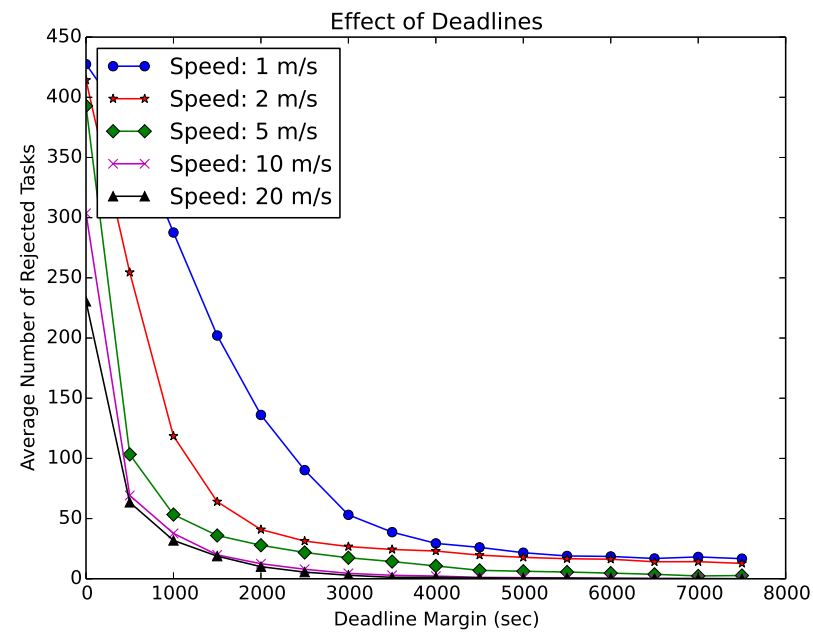

(a) Average number of rejected tasks

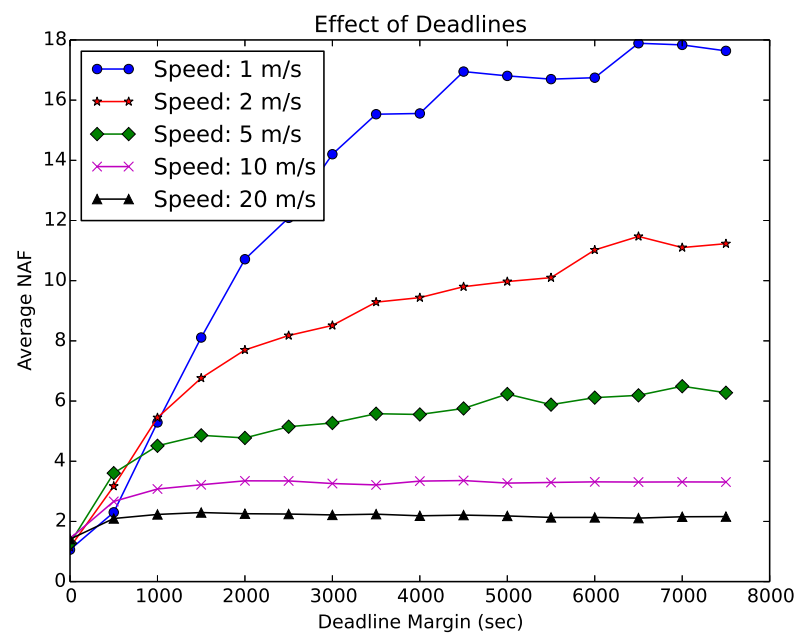

(b) Average Objective Value.

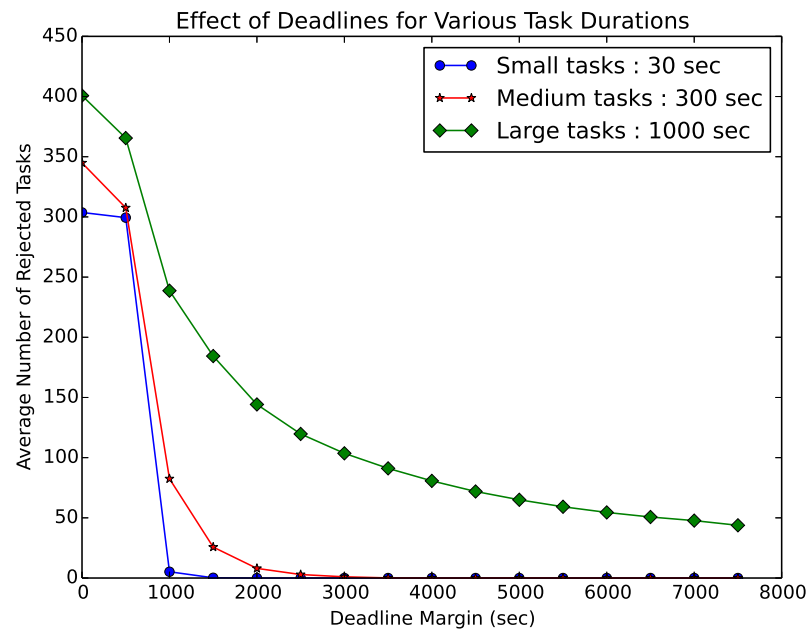

(c) Effect of deadlines on task rejections with task duration.

Figure 15: Effect of deadlines. 